\title{
Micosociología: antecedentes históricos, evolución y proyecciones
}

\author{
(Mycosociology: Historical Background, evolution and projections)
}

\author{
Viviana E. Salazar Vidal* \\ Departamento de Botánica, Facultad de Ciencias Naturales \\ y Oceanográficas,Universidad de Concepción. \\ *Autora para correspondencia: vivianasalazar@udec.cl
}

RECIBIDO:06 de Noviembre de 2016

APROBADO:21 de Noviembre de 2016

DOI: $10.22370 /$ bolmicol.2016.31.2.487

LA AUTORA DECLARA NO TENER CONFLICTO DE INTERESES

Palabras clave: Macromicetos, micosociología, comunidades fúngicas, revisión

Key words: Macromycetes, micosociology, fungal communities, review

\section{RESUMEN}

A lo largo de la historia numerosos investigadores han intentado estudiar la sociología de los hongos, también llamada micosociología que se refiere al estudio y la clasificación de las comunidades fúngicas, sus interrelaciones y su dependencia del medio. La mayoría de estos estudios han sido realizados en Europa, donde destacan los trabajos realizados por Darimont (1973) y García Bona (1977).

Son pocos los estudios de carácter micosociológico que describen la interacción de ciertos macromicetos con especies de Nothofagus de América del Sur, destacando los de Godeas et al. (1993 a, b, c) en bosques de Nothofagus de Tierra del Fuego y el de Valenzuela et al. (1998).

En esta revisión, se exponen los comienzos históricos de la micosociología, su evolución a lo largo de los años y cómo su enfoque ayuda a comprender el funcionamiento de las comunidades fúngicas. También, se destaca la importancia que tiene para nuestro país realizar estudios micosociológicos, especialmente, en bosques nativos dominados por Nothofagus.

\section{ABSTRACT}

Over time many researchers have tried to study the sociology of fungi, or mycosociology, meaning the study and classification of fungal communities, their inter-relations and their dependence on the medium. The majority of these studies have been carried out in Europe, notably the works of Darimont (1973) and García Bona (1977).

There are few mycosociological studies which describe the interaction of certain macromycetes with South American Nothofagus species. Important studies are Godeas et al. (1993 a, b, c), in Nothofagus forests in Tierra del Fuego, and Valenzuela et al. (1998). 
This review examine the historical beginnings of mycosociology, its evolution over time and how its focus helps to understand the functioning of fungal communities. The autor also stress the importance for Chile of carrying out mycosociological studies, especially in native forests dominated by Nothofagus.

\section{INTRODUCCIÓN}

En el transcurso del desarrollo de la micosociología han existido dos tendencias principales: la primera basada en estudios fitosociológicos, ha intentado incluir los macromicetos dentro de las comunidades de plantas superiores y la segunda tendencia, aceptada actualmente por la mayoría de los micólogos, sugiere la existencia de una sociología de hongos superiores independiente (Moreno \& López 1978, Kreisel \& Dörfeldt 1985).

No es adecuado incluir los macromicetos como un todo homogéneo dentro de una comunidad forestal, ya que existe una gran diversidad de estos organismos coexistiendo en un bosque, ya sea de manera saprofítica (sobre hojarasca, restos vegetales y/o de animales, excrementos), simbiótica (formando micorrizas con plantas superiores) o parasítica (Webster \& Weber 2007). Además, la especificidad de algunos macromicetos a una determinada especie o grupo de especies arbóreas, es mayor de lo que se cree, particularmente en especies micorrízicas, lo que aumenta la variedad de ambientes ecológicos donde pueden desarrollarse las comunidades fúngicas (Dighton et al. 2005).

Los hongos son uno de los grupos más diversos de organismos en la Tierra y constituyen un componente biótico fundamental de los ecosistemas terrestres (Keizer 1998, Seen-Irlet et al. 2007, Feest 2010). Son organismos muy importantes para el reciclaje de materia orgánica, valiosos actores en el ciclo biogeoquímico del carbono y desempeñan una enorme variedad de funciones esenciales para el correcto funcionamiento de los ecosistemas
(Arnolds 1992, Trappe \& Luoma 1992, Pan et al. 2008, Montoya et al. 2010).

Esta revisión se centra en el estudio de la sociología de hongos superiores, también conocidos como macromicetos o macrohongos. $\mathrm{Su}$ objetivo es resumir sus antecedentes históricos, describir su evolución y proyecciones, analizando los resultados de los principales intentos realizados por algunos micólogos para estudiar la micosociología.

\section{Importancia de la Micosociología}

La micosociología nos permite estudiar la relación que existe entre distintos grupos de hongos (micocenosis), entre los hongos y otros seres vivos (biocenosis) y con su ambiente. Los hongos se distribuyen ampliamente en el planeta, favorecidos por su rápida reproducción, pudiendo ser encontrados en diferentes hábitats, siempre que exista suficiente cantidad de agua y nutrientes disponibles y temperatura óptima para su crecimiento (Herrera \& Ulloa 2004).

Factores bióticos y abióticos determinan la fructificación de diferentes especies de hongos. Factores como la composición, edad y estructura de las formaciones vegetales determinan la composición de macromicetos, ya que las plantas constituyen los hábitats y fuente de energía para la mayoría de las especies fúngicas, las que siempre presentan algún grado de especificidad por el hospedante o el tipo de sustrato (Termorshuizen \& Schaffers 1991, Sarrionandia 2006). Respecto a la gran variación interanual que presenta la fructificación de carpóforos (Vogt et al. 1992, Straatsma et al. 2001, Martínez-Peña 2003), ésta se debe principalmente a la influencia que ejercen diversos factores meteorológicos y ecológicos (Luoma et al. 1991, Laganà et al. 2002; Baptista et al. 2010, Boddy et al. 2014).

Existe una estrecha relación entre especies vegetales y algunos macromicetos, en especial si se trata de especies micorrízicas (Egli 2011). Esto 
nos hace concluir que existe una diversidad de especies de macromicetos característica para cada tipo de vegetación. Por este motivo, la distribución de los macromicetos suele estar asociada a la cobertura vegetal y su estado fenológico, la textura del suelo y la composición de la hojarasca (SennIrlet \& Bieri 1999).

Varios autores coinciden en que las micorrizas pueden contribuir a la salud de las plantas y a su productividad al aumentar su tolerancia a diversas enfermedades y parásitos (Marschner \& Dell 1994, Maldonado \& Ramírez 1997, Miyasaka \& Habte 2003, Egli 2011). De acuerdo a Bethlenfalvay \& Linderman (1992), se estima que el 80\% de las plantas vasculares se encuentran asociadas a hongos, sin los cuales no resistirían eventos climáticos, tales como: la sequía o la falta de nutrientes en el suelo, o serían atacadas con mayor fuerza por ciertos patógenos.

Hoy en día, la micosociología adquiere una importancia particular para los hongos en peligro de extinción, ya que en muchos países ha habido una notable disminución de la cantidad de especies de macromicetos, sobre todo durante las últimas tres décadas (Dahlberg \& Mueller 2011). Esto se debe, principalmente, a la destrucción de su hábitat y cambios en la vegetación. Por lo tanto, la micosociología juega un papel importante estableciendo relaciones entre los macromicetos, la vegetación y los factores climáticos, que permiten definir patrones de diversidad y distribución para caracterizar un área geográfica determinada. El análisis de la diversidad de macromicetos ha sido utilizado para el planeamiento de áreas estratégicas de conservación, ya que poseen una alta diversidad de especies, superior a las plantas con flores y son sensibles a los cambios ambientales como los cambios en la cobertura vegetal (Falkengren-Grerup et al. 1994, Balmford et al. 2000).

Comprender cómo funcionan las comunidades fúngicas es de suma importancia para lograr un enfoque comunitario de la diversidad y distribución de los macromicetos, considerando su interdependencia con otros organismos. Si lográramos entender mejor cómo se relacionan los hongos entre sí y con otros seres vivos, podríamos predecir los lugares donde habitarán ciertas especies, si las condiciones del clima se han mantenido en el tiempo o han ido cambiando (Bünteng et al. 2012). Podríamos también conocer el grado de alteración que presentan los ecosistemas donde habitan (Tóth \& Barta 2010) y con ello contribuir a la conservación de ciertas especies.

\section{Concepto y evolución de la Micosociología}

La micosociología se refiere al estudio de las interacciones entre los componentes de las comunidades fúngicas (Kirk et al. 2008). Desarrollar la sociología de macromicetos ha sido difícil, debido a una serie de problemas propios de su naturaleza, como su carácter heterótrofo, su dependencia a un sustrato y que sus cuerpos fructíferos sólo pueden observarse en forma directa cuando se presentan condiciones adecuadas, pues no fructifican si las condiciones meteorológicas no son favorables algunos años (Moreno \& López 1978, Boddy et al. 2014).

Se sugiere que para lograr el estudio de las comunidades fúngicas es necesario tener en cuenta que lo importante es el tipo y la cantidad de micelio, así como también, el sustrato donde habita el hongo estudiado (García Bona 1976). Según su forma de vida y de alimentación, se pueden distinguir tres clases de micelio: saprófito, parásito y micorrízico, cada uno de los cuales requiere sustratos diferentes con una composición específica. García Bona (1976) entrega el siguiente ejemplo: un mismo sustrato bajo condiciones ambientales totalmente distintas, puede permitir el crecimiento de diferentes comunidades fúngicas.

Una de las obras más importantes sobre micosociología fue la realizada por Darimont (1973), 
donde el autor desarrolló un método práctico para reconocer las especies de macromicetos existentes en los bosques de Bélgica, considerando la menor o mayor abundancia de micelio de cada una de éstas, lo que marcaría la dominancia de una especie sobre otras. Asimismo, destaca el estudio realizado por García Bona (1977) para determinar los macromicetos presentes en comunidades arbóreas de Navarra. Se han realizado otros trabajos en esta línea de investigación, pero la mayoría de ellos no ha tenido éxito por carecer de un método apropiado de estudio y no contar con procedimientos adecuados para analizar los datos obtenidos.

Darimont estableció un sistema formal de nomenclatura micosociológica para aplicar a los datos que había recopilado en bosques de Bélgica. De este modo, el autor introdujo un sistema separado para nombrar a los hongos en el que la unidad básica fue denominada sociomicete (nombre que termina en "ecium", por ejemplo, Amanitecium muscariae, lo que es más o menos similar a una asociación) que se agrupan (en orden ascendente) como "alianzas" ("ecion"; Boletecion scabri) "órdenes" ("ecia": Boleto-Amanitecia), y "clases" ("ecea"; Cortinario-Boletacea).

Darimont mencionó en su obra los intentos realizados por cinco grandes micólogos para entender la sociología de los macromicetos en Europa: Gilbert (1929), Haas (1933), Höfler (1937), Leischner-Siska (1939) y Pirk (1948).

Gilbert (1929): Propuso una escala de cuatro grados de abundancia: RR (esporádica), R (ocasional), C (frecuente) y CC (abundante), sin embargo, esta escala tenía un carácter de síntesis y no de análisis. Este autor rechazó considerar la sociología (agrupaciones de carpóforos) como un carácter relevante.

Haas (1933): Fue uno de los primeros micólogos en estudiar la micosociología y sus métodos todavía son empleados. Propuso una escala de abundancia de seis grados: + (una sola localidad), 1 (algunas localidades poco numerosas), 2 (muy dispersas), 3 (dispersión irregular), 4 (localidades numerosas, extendidas en el lugar) y 5 (en masa, abundante por toda la zona). En esta escala la "localidad" es el lugar o punto donde se produce la fructificación. La escala es buena, pero demasiado amplia desde el punto de vista práctico. Este autor consideró importante la sociabilidad y la estudió en una escala de cinco grados basada en la de Braun-Blanquet (1928).

Höfler (1937): Estableció las líneas rectoras de la sociología de los hongos. Introdujo el peso de los carpóforos como medida cuantitativa para evaluar la abundancia. Intentó inferir la cantidad de micelio utilizando el número y tamaño de los carpóforos $(\mathrm{CF})$, para lo cual emplea la siguiente fórmula: $\mathrm{P}=\mathrm{N}^{\circ}$ de $\mathrm{CF}$ x peso de los CF. Este autor se basó en la escala de seis grados de Haas y no consideró la sociabilidad.

Leischner-Siska (1939): Utilizó la escala combinada de seis grados de abundancia-dominancia de Braun-Blanquet (1928), sin embargo, el mismo autor reconoció que en la práctica suelen utilizarse los dos primeros grados. Consideró importante la sociabilidad y la estudió en una escala de cinco grados (Braun-Blanquet 1928), empleando áreas de $100 \mathrm{~m} 2$. Leischner-Siska, relacionó el número de especies de hongos presentes en el área con variaciones en la temperatura y las precipitaciones.

Pirk (1948): Su método se basó en el recuento de carpóforos, agrupándolos después según una escala de seis grados: + (1 carpóforo), 1 (2-5 carpóforos), 2 (6-10 carpóforos), 3 (11-20 carpóforos), 4 (21-50 carpóforos) y 5 (más de 50 carpóforos). Esta escala suele ser poco práctica en áreas grandes, pero muy útil para áreas pequeñas.

Otros autores que han realizado estudios micosociológicos relevantes, ya sea aplicando los métodos propuestos previamente o modificándolos 
son: Hueck (1953), Guminska (1962), Lisiewska (1963), Kuulo Kalamees (1968), Thoen (1970), Chevassut \& Moussain (1973), Darimont (1973), Losa Quintana (1974), García Bona (1977), Godeas et al. (1993 a, b, c), Valenzuela et al. (1998), entre otros.

Hueck (1953): Hizo una revisión profunda de la metodología micosociológica, estudiando las comunidades de hongos por separado, por razones tanto prácticas como teóricas y sugiriendo un método para estudiar la sociología de los macromicetos, basándose en la aparición de carpóforos al azar que era la única información disponible para realizar estudios en el campo.

Darimont (1973): Consideraba de gran importancia la fisonomía del carpóforo (hongo agaricoide, corticoide, etc.) y la sociabilidad (forma en la que se desarrollan los cuerpos fructíferos, es decir, aislados, en grupos, en masa, etc.). Ambas características pueden estar condicionadas por el sustrato y/o por las condiciones ambientales. Además, propuso algunas pautas metodológicas para trabajar en micosociología, partiendo de la idea que los hongos son totalmente distintos a otros organismos.

Según este autor, los distintos aspectos a considerar en un ecosistema fúngico son:

1. Buscar las localidades micológicas adecuadas (micotopos fáciles de diferenciar). Ej. Micotopos terrestres, lignícolas, briofíticos, accidentales. Estos micotopos están condicionados por la vegetación predominante.

2. Analizar cada uno de estos micotopos con su diversidad fúngica, dentro de cada asociación (considerar la fisionomía y sociabilidad). Hacer una lista de hongos.

3. Síntesis de los datos recogidos (técnicas a seguir basadas en Braun-Blanquet 1928). Ordenar la información recopilada.
Si bien el método de Darimont puede ser útil para analizar las comunidades fúngicas, hay ocasiones donde puede ser difícil de aplicar, por ejemplo, en grandes áreas. En este último caso, este método requiere mejorar algunos aspectos metodológicos tales como, el modo de selección de las áreas donde se realizarán inventarios fúngicos y la toma de datos.

García Bona (1977): Considera las comunidades fúngicas como algo independiente y defiende la existencia de una sociología autónoma de hongos superiores. Estudió la relación de macromicetos con las principales comunidades boscosas de Navarra (España), siendo uno de los pioneros en abordar el campo de la micosociología en España.

En América del Sur son escasos los estudios de carácter micosociológico realizados a la fecha. Singer \& Morello (1960) fueron los primeros en intentar establecer la relación existente entre macromicetos ectomicorrícicos (Agaricales) y bosques de Nothofagus. Singer \& Moser (1965), basándose en observaciones personales consiguieron estudiar la micoflora estacional de comunidades de Nothofagus en la Cordillera Pelada ubicada en la Región de los Ríos. Singer (1971) fue quien logró realizar un estudio micosociológico y de sucesión de macromicetos en bosque mixto de Coigüe ( $N o$ thofagus dombeyi) y Ciprés de la Cordillera (Austrocedrus chilensis). Posteriormente, empleando la clasificación de Orlos (1965), Wright (1988) logró relacionar algunos macromicetos a distintos bosques de Nothofagus. Asimismo, Garrido (1988) hizo un estudio taxonómico y ecológico sobre macromicetos que fructifican en bosques de Nothofagus, comprendidos entre las regiones del Maule y Biobío.

Posteriormente, Godeas et al. (1993 a, b, c) determinaron una serie de características de las comunidades de macromicetos, incluidos los Agaricales, que fructifican en bosques de Nothofagus 
de Tierra del Fuego y Valenzuela et al. (1998) realizaron un estudio comparativo de tipo micosociológico y taxonómico de los Agaricales s. l. que fructifican en bosques de Nothofagus y plantaciones de Pinus radiata que prosperan a nivel de la Cordillera de la Costa, Depresión Intermedia y Cordillera de los Andes de la X Región de Chile.

\section{Métodos utilizados para el estudio de la Mico- sociología}

Actualmente, el avance de técnicas moleculares permite determinar taxonómicamente $\mathrm{y}$ cuantificar el micelio por unidad de volumen de suelo, de una manera más fácil, rápida y confiable, expresada en forma de biomasa total de micelio de toda la comunidad fúngica o cuantificación de una especie fúngica particular (Rodríguez-Tovar et al. 2004, Suz et al. 2006, Parladé et al. 2007, Hortal et al. 2008). Sin embargo, no siempre existe una relación directa entre la cantidad de micelio o micorrizas y la producción de carpóforos (Gardes \& Bruns 1996), por lo que gran parte de los estudios micosociológicos todavía siguen basándose en la determinación y cuantificación de los carpóforos, siendo su recolección fundamental para realizar identificaciones moleculares (Osmundson et al. 2013).

El estudio micosociológico a partir de carpóforos presenta la dificultad de que los muestreos deben coincidir con el momento de fructificación de las especies y no todas las especies fructifican cada año, además su desarrollo suele ser muy breve (Ratowsky 2007, Body et al. 2014). Por otra parte, hay especies cuyas fructificaciones aparecen de forma regular cada año, mientras que en otros casos son muy raras, pudiendo fructificar cada 10 años o más (Oria de Rueda 2007). Estas circunstancias complican los muestreos, siendo necesarios los inventarios semanales o quincenales en épocas productivas, durante varios años. Otra forma de estudiar la micocenosis se basa en los muestreos y de- terminación de morfotipos micorrícicos (de Román $\&$ de Miguel 2005).

Las comunidades de hongos han sido poco estudiadas, debido a las dificultades en su determinación y también porque los inventarios realizados en un solo momento no entregan una idea correcta de su composición (Hawsworth 1991). Asimismo, los procesos de toma de inventarios fúngicos son complejos de realizar. La recolección en el campo se hace utilizando distintos protocolos de muestreo, a través de la toma de fotografías in situ, descripción de los ejemplares colectados, secado, empacado e identificación microscópica. El inventario de hongos apropiadamente colectado y mantenido, genera información de fácil acceso y comprensión para todo público, incluyendo aquel que no es especialista en el tema.

En los inventarios de las comunidades de macromicetos hay que investigar detenidamente superficies de $100 \mathrm{~m}^{2}$ como mínimo. Tal como indica Höfler (1954), esta extensión todavía es demasiado pequeña para incluir la lista total de especies de la asociación, por lo que deben anotarse también las especies que se presenten dentro del mismo bosque fuera de la superficie delimitada.

Moreno \& López (1978) plantean como primera dificultad para un estudio de la sociología de macromicetos, la elección de una metodología adecuada y coherente, ya que la casi obligatoriedad de trabajar con carpóforos, como la única parte observable del hongo, hace inútiles la mayoría de los índices clásicos en fitosociología. Como consecuencia de lo expuesto anteriormente, estos autores consideran más importante lo siguiente:

1) La elección cuidadosa del medio ecológico, evitando la mezcla de medios distintos. En ocasiones hay que separar dentro de un mismo bosque las distintas especies arbóreas para evitar incurrir en falta de homogeneidad. 
2) La simplificación de índices fitosociológicos que deben quedar reducidos a la abundancia-dominancia según una escala resumida. Los otros índices, como sociabilidad, etc., parecen no aconsejables al estar refiriéndose a cuerpos fructíferos y obteniendo por ello poca o nula información sobre la disposición de los micelios.

3) La gran influencia de los medios climatológicos en la fructificación de los hongos hace imprescindible el estudio de cada localidad en las cuatro estaciones del año y durante un período extenso. Sólo así se podrá tener la seguridad de haber registrado todas las especies y además, con su índice correcto.

4) De esta manera, parece más sencillo el estudio de la micosociología de macromicetos, que es independiente de las plantas superiores. Con respecto a la terminología, parece más adecuado no introducir nuevas terminaciones y raíces.

Finalmente, Smith et al. (2002) señalan que un inventario continuo usando parcelas permanentes, aporta más información sobre la diversidad de carpóforos de un ecosistema, que el inventario de nuevas áreas (parcelas temporales). Para mayor información sobre algunos métodos idóneos para realizar estudios ecológicos con macromicetos se sugiere revisar: Mueller et al. (2004), Feest (2006), entre otros.

\section{¿Cómo abordar la Micosociología en Chile?}

En nuestro país son pocos los estudios ecológicos de macromicetos realizados, por lo que existe un limitado conocimiento sobre la riqueza y distribución de sus especies (Mancilla et al. 2008). La diversidad de macromicetos en Chile está formada por especies nativas e introducidas, existiendo una micoflora para Chile central y otra para Chile austral (Lazo 2001). Esta distribución se debe principalmente a las diferencias climáticas, Lazo (2001) propone que la abundancia o escasez de macromicetos está relacionada con la precipitación pluvial del lugar siendo más diversos en los bosques australes (Gamundi \& Horak 1993).

Debido a la escasez de estudios micosociológicos, a excepción del realizado por Valenzuela et al. (1998) y otros autores mencionados anteriormente, la autora de esta revisión sugiere hacer un inventario de macromicetos por región a lo largo de Chile. De esta manera, se conocería mejor la diversidad de macrohongos presentes en ecosistemas terrestres de nuestro país, así como también, los lugares exactos donde se distribuyen las especies que actualmente están consideradas en peligro, de modo que podamos ayudar a su conservación. Una posibilidad de avance es diseñar y llevar a cabo inventarios en bosques de Nothofagus por las siguientes razones: 1) La mayor parte de macromicetos ectomicorrícicos nativos se encuentran asociados a árboles de este género, al igual que algunas especies parásitas biotróficas como los Digüeñes (Cyttaria spp.); 2) Muchos árboles de este género se encuentran en peligro, como es el caso del Hualo (Nothofagus glauca) y el Ruil (Nothofagus alessandrii) que está clasificado en peligro de extinción y; 3) El género Nothofagus ha sido designado como clave para la fitogeografía, debido a su especial distribución disyunta (Darlington 1965, Van Steenis 1972).

Otras consideraciones importantes que pueden ser aplicadas en un estudio micosociológico en nuestro país son las que plantean Dighton et al. (2005), quienes indican que lo que interesa es conocer las especies fúngicas existentes en un lugar determinado y tener una idea de la mayor o menor abundancia de micelio de cada especie, lo que marcaría la dominancia de una de ellas. Cuantificando los carpóforos de cada especie podemos tener una idea de la diversidad que hay en un bos- 
que, sin embargo, no estaríamos considerando los micelios existentes que no producen carpóforos, lo que hace que el estudio no sea representativo. Por lo tanto, la estacionalidad o periodicidad en la producción de cuerpos fructíferos visibles es variable, ya que en algunos casos los carpóforos pueden durar muy poco tiempo, mientras que en otros una misma especie puede no producir carpóforos durante un año o una década. Por ejemplo, en un estudio de 21 años de parcelas en Suiza, Straatsma et al. (2001) encontraron cuerpos fructíferos de 408 especies. Sin embargo, el número registrado en cada año, incluso después de repetidas visitas, oscilaba entre 18 y 194, con 19 especies no encontradas el último año de estudio.

Si se desea obtener un inventario total, al examinar los macromicetos presentes en un sitio determinado también debemos considerar el número de nichos ecológicos diferentes existentes (Mueller et al. 2004). Estas diferencias pueden atribuirse al menos en parte a la naturaleza heterotrófica de los hongos, pues debido a su capacidad para especializarse en ciertos sustratos, huéspedes o plantas asociadas, es que los macromicetos presentan una mayor diferenciación de nichos que las plantas, permitiendo así que existan muchas más especies (Barkman 1987, Venturella et al. 2011). Por lo tanto, un examen exhaustivo de los hongos en un sitio requiere el estudio de una muy amplia gama de hábitats, muchos de las cuales requieren diferentes técnicas, así como las aportaciones de micólogos con diferentes habilidades (Hawksworth 2001).

Uno de los métodos para realizar estudios micosociológicos que es considerado interesante es el propuesto por Nespiak (1959), quien estudió la composición específica, frecuencia de carpóforos y sociabilidad de los hongos en espacios determinados durante un periodo razonable de tiempo. Además, consideró importante para su investigación medir al mismo tiempo factores microcli- máticos determinantes, tales como: temperatura máxima y mínima, precipitaciones y evaporación. El método descrito por este autor es válido, pero necesita algunas modificaciones, tales como: a) medir y pesar los carpóforos con el fin de establecer su abundancia, b) que los espacios estudiados tengan una extensión de 100 a $200 \mathrm{~m} 2$, c) que la realización del inventario tenga una duración igual o superior a los tres años como lo sugiere (Sarrionandia 2006) para evitar que queden demasiadas especies de macromicetos sin contabilizar a causa del factor presencia/ausencia de carpóforos por año.

\section{CONCLUSIONES}

La micosociología es importante para conocer el ecosistema terrestre y entender las distintas relaciones que existen entre las comunidades fúngicas y otros componentes bióticos. Dentro de los métodos actuales para su estudio se encuentran técnicas moleculares que permiten determinar ciertas especies a partir de su micelio de una forma más fácil y rápida, aunque siguen predominando los métodos basados en la recolección de carpóforos.

Conocer la diversidad taxonómica y distribución de los macromicetos presentes en un área geográfica, permitiría ayudar a la conservación de algunas especies y sería un indicador para detectar el grado de alteración de un ecosistema. Además, ayudaría a comprender los mecanismos de interacción entre las comunidades fúngicas y los de plantas y/o animales con que comparten un ecosistema.

Es de suma importancia que en nuestro país se comiencen a realizar inventarios de macromicetos con un enfoque micosociológico en bosques nativos dominados por Nothofagus, ya que esto podría significar una contribución a la hora de ir en búsqueda de especies de macrohongos en peligro o difíciles de encontrar. 
Micosociología: Antecedentes históricos, evolución y proyecciones - Salazar V.

Finalmente, a la hora de llevar a cabo un estudio micosociológico se recomienda utilizar métodos modernos que combinen la observación en terreno, la caracterización morfológica y la diversidad.

\section{AGRADECIMIENTOS}

La autora agradece a los colegas Mario Ramos y Carolina Rose por su ayuda y recomendaciones.

\section{REFERENCIAS}

Arnolds, E. 1992. The analysis and classification of fungal communities with special reference to macrofungi. In Fungi in vegetation science (pp. 7-47). Springer Netherlands.

Barkman, J., Moravec, J. \& S. Rauschert. 1986. Code of phytosociological nomenclature. Vegetatio 67: $145-195$.

Barkman, J. 1987. Methods and results of mycocoenological research in the Netherlands. p. 7-38. In: G. Pacioni (ed.) Studies on fungal communities. University ofl'Aquila, Italy.

Balmford, A., Lyon, A. \& R. Long. 2000. Testing the higher-taxon approach to conservation planning in a megadiverse group: the macrofungi, Biological Conservation. 93(2):209-217.

Baptista, P., Martins, A., Tavares, R. \& T. LinoNeto. 2010. Diversity and fruiting pattern of macrofungi associated with chesnut (Castanea sativa) in the Trás-os-Montes región (Northeast Portugal), Fungal Ecology 2010; 3(1): 9-19.

Bethlenfalvay, G. J. \& J. Linderman. 1992. Mycorrhizae and crop productivity. USA: Horticultural Crops Research Laboratory, USDA-ARS.

Boddy, L., Bunteng, U., Egli, S., Gange, A.C., Heegaard, E., Kirk, P.M., Mohammad, A. \& H. Kauserud 2014. Climate variation effects on fungal fruiting. Fungal Ecology 10: 20-33.

Braun-Blanquet, J. 1928. Pflanzensoziologische Grundzüge der Vegetationskunde. In: Biologische Studienbücher (W. SCHÖNICHER, Ed.), 7, 330 ff. Berlin, Springer.
Bünteng, U., Kauserud, H. \& S. Egli. 2012. Linking climate variability to mushroom productivity and phenology. Front Ecol Environ 10(1): 14-19. Chevassut \& Moussain. 1973. Macroflore fongique du Pin Maritime (Montpellier). Bull. Soc. Mycol. Fr., 89 (2): 229-251.

Dahlberg, A. \& G.M. Mueller. 2011. Applying IUCN red-listing criteria for assessing and reporting on the conservation status of fungal species. Fungal Ecol 4:147-162.

Darimont, F. 1973. Recherches mycosociologiques dans les forets de Haute-Belgique. Essai sur les fondements de la sociologie des champignons supérieurs. Inst. R. des Sc. Nat. de Belgique, Mémoire 170.

Darlington, P. J. 1965. "Geographic history of Nothofagus". En: Biogeography of the Southerend of the world. Harvard Univ. Press, pp. 140-148.

De Román, M. \& A. De Miguel. 2005. Post-fire, seasonal and annual dynamics of the ectomycorrhizal community in a Quercus ilex L. forest over a 3-year period. Mycorrhiza 15:471-482

Dighton, J., White, J. \& P. Oudemans. 2005. The Fungal Community: Its Organization and Role in the Ecosystem, 3rd ed.. CRC Press. 890 pp.

Egli, S. 2011.Mycorrhizal mushroom diversity and productivity - an indicator of forest health?. Annals of forest Sciences 68: 81-88.

Falkengren-Grerup, U., Rühling, Ä. \& G. Tyler. 1994. Effects of phosphorus application on vascu- 
lar plants and macrofungi in an acid beech forest soil, The Science of the Total Environment 151(2): 125-130. Feest, A. 2006. Establishing baseline indices for the quality of the biodiversity of restored hábitats using a standardized sampling process. Restoration Ecology 14, 112-122.

Feest, A., Aldred, T. \& K. Jedamzik. 2010. Biodiversity quality: a paradigm for biodiversity. Ecol. Ind. 10:1077-1082.

Gamundí, I. \& E. Horak. 1993. Hongos de los bosques Andino-Patagónicos. Vázquez Mazzini Editores, Buenos Aires. 141 pp.

Gardes, M. \& T. Bruns. 1996. Community structure of ectomycorrhizal fungi in a Pinus muricata forest: above and below-ground views. Can. J. Bot. 74: 1572-1583.

García Bona, L. 1976. Introducción a la Micosociología. Revisión de los principales métodos empleados hasta nuestros días). Anales de la Estación Experimental del Aula Dei, 13 (3-4).

García Bona, L. 1977. Estudio micoecológico de las principales comunidades arbóreas de la Navarra Alta y Media (Tesis Doctoral inédita). Facultad de Ciencias, Universidad de Navarra, Pamplona, España.

Garrido, N. 1988. Agaricales s.l. und ihre in den Nothofagus-wäldern mittelchiles. Bibliotheca Mycologica, Band 120, J. Cramer Yaduz, 528 pp.

Gilbert, E. 1929. La mycologie sur le terrain. Le François. In-12 broché. 188 pp.

Godeas, A., Arambarri, A. \& I. Gamundi. 1993a. Micosociología en los bosques de Nothofagus de Tierra del Fuego I. Diversidad, abundancia y fenología. Anales Academia Nacional de Ciencias Exactas Físicas y Naturales 45: 291-302.

Godeas, A., Arambarri, A. \& I. Gamundi 1993b. Micosociología en los bosques de Nothofagus de Tierra del Fuego II. Importancia relativa de las dis- tintas especies de macromicetes. Anales Academia Nacional de Ciencias Exactas Físicas y Naturales 45: 303-311.

Godeas, A., Arambarri, A. \& I. Gamundi. 1993c. Micosociología en los bosques de Nothofagus de Tierra del Fuego III. Análisis de las comunidades de macromicetes. Anales Academia Nacional de Ciencias Exactas Físicas y Naturales, Buenos Aires 45: 313-318.

Guminska. 1962. The fungi of the beach forests of Rabsztyn and Maciejawa. Monogr. Bot. 13: 3-38. Haas, H. 1933. Die bodenbewohnenden Großpilze in den Waldformationen einiger Gebiete von Wurttemberg. Beihefte zum Botanischen Zentralblatt 50: 35-34.

Hawsworth, D.1991. The fungal dimension of biodiversity: Magnitude, significance and conservation. Mycological Research 95: 641-655.

Hawksworth, D. 2001. The magnitude of fungal diversity: the 1.5 million species estimate revisited. Mycol. Res. 105:1422-1432.

Herrera, T. \& M. Ulloa. 2004. El reino de los hongos, Editorial Progreso S.A., México D.F., pp $25-$ 28.

Höfler, K. 1937. Pilzsoziologie. Ber. Deutsch. bot. Ges. 55; 606-622.

Hofler, K. 1954. Ueber Pilzaspekte. Vegetatio 5-6: 373-380.

Hortal, S., Pera, J. \& J. Parladé. 2008. Tracking mycorrhizas and extraradical mycelium of the edible fungus Lactarius deliciosus under competition with Rhizopogon spp. Mycorrhiza 18: 69-77.

Hueck, H. J. 1953. Myco-sociological methods of investigation. Vegetatio 4: 84-101.

Kalamees, K. 1968. Mycocoenological methods based on investigations in the Estonian forests. Acta Mycol. 4 (2): 327-335. 
Kirk, P.M., Cannon, P.F., Minter, D.W. \& J.A. Stalpers. 2008. Ainsworth and Bisby's Dictionary of Fungi, 10th edition. Oxford Universitiy Press.

Keizer, G. 1998. The complete encyclopaedia of mushrooms. Rebo Publishers Netherland.

Kreisel, H. \& H. Dorfelt. 1985. Pilzsoziologie. p. 67-95. In: Michael, Hennig, and Kreisel (eds.) Handb. Pilzfr. 4 (3. Aufl.) Gustav Fischer, Jena.

Laganà, A., Angiolini, C., Loppi, E., Salerni, E., Perini, C., Barluzzi, C. \& V. De Dominicis. 2002. Periodicity, fluctuations and succesions of macrofungi in fir forests (Abies alba Miller) in Tuscany, Italy. For. Ecol. Manag. 169: 187-202.

Lazo, W. 2001. Hongos de Chile. Atlas Micológico. Facultad de Ciencias de la Universidad de Chile. Editorial Universitaria, Chile. 230 pp.

Leischner-Siska, E. 1939. Zur Soziologie und Okologie der hoheren Pilze Untersuchung der Pilzvegetation in der Umgebung von Salzburg wahrend des Maximalaspektes 1937. Beih Bot Centralbl, B 59: 359-429.

Lisiewska, M. 1963. Higher fungi of forest associations of the beech forest near Szczecin. Monogr. Bot. 15: 77-151.

Losa Quintana, J.M. 1974. Macromicetes del bosque de Quercus robur L. Anales del Jardín Botánico de Madrid 31(1): 185-197.

Luoma, D.L., Frenkel, R.E. \& J.M. Trappe. 1991. Fruiting of hypogeous fungi in Oregon Douglas-fir forests: seasonal and habitat variation. Mycologia 83: 335-353.

Maldonado J. \& G. Ramírez. 1997. Efecto de la inoculación con hongos micorrizógenos en almácigos de café (coffea arábiga) Variedad Colombia. Universidad Nacional de Colombia. Tesis, Facultad de Ciencias Agropecuarias, Medellín, pp. 3 - 83.
Mancilla, V., Henríquez, J. \& J. Vera. 2008. Biodiversidad de macrohongos de la Reserva Nacional Magallanes. Anales del Instituto de la Patagonia, 36(1), 35-44.

Martínez-Peña, F. 2003. Producción y aprovechamiento de Boletus edulis Bull.: Fr. en un bosque de Pinus sylvestris L. Consejería de Medio Ambiente. Junta de Castila y León, 134 pp.

Marschner, H. \& B. Dell. 1994. Nutrient uptake in mycorrhizal symbiosis. Plant and soil 159: $89-$ 102.

Miyasaka, S. \& M. Habte. 2003. Plant mecanisms and mycorrhizal symbiosis to increase phosphorus uptake efficiency. Journal Series Nro. 4468, College of tropical Agriculture and Human resources, Hawaii, Honolulu, pp. 1101 - 1133.

Montoya, S., Gallegos, J., Sucerquía, A., Peláez, B., Betancourt, O. y D. Arías. 2010. Macromicetos observados en bosques del departamento de Caldas: su influencia en el equilibrio y la conservación de la biodiversidad. Boletín Científico. Museo de Historia Natural. 14(2): 57-73.

Moreno, G. \& G. López. 1978. Sobre la sociología de Macromycetes. Las comunidades de abedulares de la Sierra de Guadarrama (Melico-Betuletum celtibericae). Anal. Inst. Bot. Cavanilles 34(2):439-465.

Nespiak, A. 1959. Studia nad udzialem grzybow kapeluszowych w zespolach lesnych na terenie Bialowieskiego Parku Narodowego (The investigations on the character of the correlations between the higher fungi and forest associations in the National Park of Bialowieza). Monogr. Bot. 8: 3-141.

Mueller, G.M., Schmit, J.P., Huhndorf, S.M., O'Dell, T.E., Lodge, D.J., Leacock, P.R., Ryvarden, L., Mata, M.M., Umaña, L., Wu, Q.-X. \& D. Czederpiltz. 2004. Measuring and monitoring diversity of terrestrial and Lignicolous macrofungi: 
recommended protocols for sampling macrofungi. In Biodiversity of Fungi: Inventory and Monitoring Methods, Mueller, G.M., Bills, G.F., Foster, M.S., Eds. New York, Academic Press, pp. 123-168

Oria de Rueda J. 2007. Conservación y gestión de las tejedas en la Comunidad Autónoma de Castilla y León. En: El tejo en el Mediterráneo Occidental: Ministerio de Medio Ambiente. Generalitat Valenciana: 111-118.

Pan, H., Yu, J.F., Wu, Y.M., Zhang, T.Y. \& H.F. Wang. 2008. Diversity analysis of soil dematiaceous hyphomycetes from the Yellow River source area. J Zhejiang Univ Sci B. 6:64-69.

Parladé, J., Hortal, S., Pera, J. \& L. Galipienso. 2007. Quantitative detection of Lactarius deliciosus extraradical soil mycelium by real-time PCR and its application in the study of fungal persistence and interspecific competition. J. Biotechnol. 128: 14-23.

Pirk, W. 1948. Zur soziologie der Pilze im Querceto-Carpinetum. Zeitschr. Pilzk. N.F. 21: 11-20.

Ratowsky, D. 2007. Visualising Macrofungal Species Assemblage Compositions Using Canonical Discriminant Analysis. Australasian Mycologist 26(2-3), 75-85.

Rodríguez-Tovar, A., Valdés, M. \& B. Xoconostle-Cásarez. 2004. Ecología molecular de los hongos ectomicorrízicos. Revista Fitotecnia Mexicana. Vol. 27 (3):267 - 278.

Sarrionandia, E. 2006. AE (2006) Estudio de las micocenosis de los macromicetos de los encinares del País Vasco. Tesis Doctoral. Universidad del País Vasco. 325 pp.

Senn-Irlet, B. \& G. Bieri. 1999. Sporocarp succesion of soil-inhabiting macrofungi in an autochthonous subalpine Norway spruce forest of Switzer- land, Forest Ecology and Management. (1999); 124 (2-3): 169-175.

Seen-Irlet, B., Heilmann-Clausen, J., Genney, D. \& A. Dahlberg. 2007. Guidance for the conservation of mushrooms in Europe. Convention on the conservation of European wildlife and natural habitats. 27th meeting, Strasbourg, 26-29 November, 34p.

Singer, R. \& J. Morello. 1960. Ectotrophic forest tree mycorrhizae and forest communities. Ecology 41: 549-555.

Singer, R. \& M. Moser. 1965. Forest mycology and forest communities in South America. The early fall aspect of the mycoflora of the Cordillera Pelada (Chile). Mycopathologia et Mycologia Applicata 26: 129-191.

Singer, R. 1971. Forest mycology and communities in South America II. Mycorrhiza sociology and fungus succession in the Nothofagus-Austrocedrus chilensis wood of Patagonia. En: Hacskaylor R (ed) Mycorrhizae - USDA Forest Service. 1189: 1-255. Smarda, F. 1973. Die Pilzgesellschaften Einiger Fichtenwälder Mährens - Acta Sc. Nat. Brno 7(8): $1-44$.

Smith, J., Molina, R., Huso, M., Luoma, D., Mckay, D., Castellano, M.A., Lebel, T. \& Y. Valachovic. 2002. Species richness, abundance, and composition of hypogeous and epigeous ectomycorrhizal fungal sporocarps in young, rotation-age, and old-growth stands of Douglas-fir (Pseudotsuga menziesii) in the Cascade Range of Oregon, USA. Can. J. Bot. 80: 186-204.

Straatsma, G., Ayer, F.\& S. Egli. 2001. Species richness, abundance, and phenology of fungal fruit bodies over 21 years in a Swiss forest plot. Mycol. Res. 105: 515-523.

Suz, L.M., Martín, M.P. \& C. Colinas. 2006. De- 
tection of Tuber melanosporum DNA in soil. FEMS Microbiol. Lett. 254: 251-257.

Thoen, D. 1970. Etude mycosociologique de quelques associations forestiéres des distriets picardo-

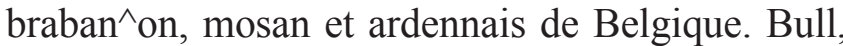
des Recherches agronomiques de Gembloux, V, núm. 1-2: 309-326.

Osmundson, T. W, Robert, V. A., Schoch, C. L., Baker, L. J., Smith, A., Robich, G., Mizzan, L. \& M. M. Garbelotto. 2013. Filling gaps in biodiversity knowledge for macrofungi: contributions and assessment of an herbarium collection DNA barcode sequencing project. PLoS ONE 8(4): e62419. doi:10.1371/journal.pone.0062419

Trappe, J.M. \& D. Luoma. 1992. The ties that bind: fungi in ecosystems. In: Carroll, G.C., Wicklow, D.T. (Ed.), The fungal community-its organization and role in the ecosystem. Marcel Dekker, New York, pp. 17-27.

Termorshuizen, A. \& A. P. Schaffers. 1991. The decline of carpophores of ectomycorrhizal fungi in stands of Pinus sylvestris L. in The Netherlands: possible causes. Nova Hedwigia 53: 267-289.

Tóth, B.\& Z. Barta. 2010. Ecological studies of ectomycorrhizal fungi: an analysis of survey methods. Fungal Divers., 45, 3-19.
Valenzuela, E., Moreno, G., Garnica, S. \& C. Ramírez. 1998. Micosociología en bosques nativos de Nothofagus y plantaciones de Pinus radiata en la X Región de Chile: diversidad y rol ecológico. Revista Chilena de Historia Natural 71: 133-146.

Van Steenis, J. 1972. "Nothofagus. key genus to plant geography". In: Taxonomy, phytogeography and Evolution. Academic Press, pp. 274-289.

Venturella, G., Altobelli, E., Bernicchia, A., Di Piazza, S., Donnini, D., Gargano, M.L., Gorjón, S.P., Granito, V.M., Lantieri, A., Lunghini, D., et al. 2011. Fungal biodiversity and in situ conservation in Italy. Plant Biosystems, 145(4): 950-957.

Vogt, K.A., Bloomfield, J., Ammirati, J.F. \& S.R. Ammirati. 1992. Sporocarp production by basidiomycetes, with emphasis on forest ecosystems. In: Carroll, G.C., Wicklow, D.T. (Ed.), The fungal community: Its organization and role in the ecosystem. Marcel Dekker, Inc., New York, pp. 563-581.

Webster, J. \& R. Weber. 2007. Introduction to Fungi. Cambridge University Press, New York. $841 \mathrm{pp}$.

Wright, J. 1988. Interrelaciones entre macromycetes (Fungi) y Nothofagus. Academia Nacional de Ciencias Exactas y Naturales 4: 135-152. 